\title{
Outage Probability and Normalized SINR-Based Power Allocation over Rician Fading Channels
}

\author{
Danh H. Ho and T. Aaron Gulliver \\ Department of Electrical and Computer Engineering, University of Victoria, PO Box 1700, STN CSC, Victoria, British Columbia, \\ Canada V8W 2 Y2 \\ Correspondence should be addressed to Danh H. Ho; danhho@uvic.ca
}

Received 20 March 2020; Revised 13 August 2020; Accepted 29 August 2020; Published 8 October 2020

Academic Editor: Pavlos I. Lazaridis

Copyright (c) 2020 Danh H. Ho and T. Aaron Gulliver. This is an open access article distributed under the Creative Commons Attribution License, which permits unrestricted use, distribution, and reproduction in any medium, provided the original work is properly cited.

\begin{abstract}
This paper considers power allocation in cellular networks over Rician fading channels. The goal is to improve the power consumption and energy efficiency as well as satisfy as many users as possible subject to user outage probability and normalized signal to interference plus noise ratio (SINR) constraints. The exact outage probability over Rician fading channels is determined using the moment-generating function (MGF). Further, upper and lower bounds on the outage probability are derived. These are used to characterize the relationship between outage probability and normalized SINR in Rician fading channels. Power allocation algorithms for power minimization and energy efficiency are proposed. Simulation results are presented to compare the performance of the proposed schemes with existing methods in terms of power consumption, throughput, energy efficiency, outage probability, and number of unsatisfied users.
\end{abstract}

\section{Introduction}

The Internet of things (IoT) is creating massive numbers of wireless connections among machines and devices resulting in significant demands on information and communication technology (ICT) and in particular wireless communication systems [1]. IoT applications include smart grids, intelligent vehicular networks, industrial automation, smart healthcare, and smart cities $[2,3]$. There are many challenges to deploying IoT networks, one of which is the energy supply of IoT devices [4]. Many IoT devices are powered by batteries with limited energy [5], and it can be very costly or impractical to replace them regularly, especially when the devices are deployed in harsh environments or embedded in human bodies [6]. Thus, energy efficiency is important for IoT devices.

While communication traffic and the number of users have increased significantly, reducing the overall power consumption of the network is a key goal. The main reasons are to reduce energy consumption costs for operators and pro- long battery life for users. A goal of 5G and beyond communication systems is better spectrum utilization and energy efficiency [7] to provide higher transmission rates and resource utilization compared to current systems $[8,9]$. Thus, power allocation is considered in this paper to reduce system power consumption, increase efficiency, and satisfy as many users as possible.

Power allocation for energy efficiency in cellular networks has previously been investigated. Target signal to interference plus noise ratio (SINR) tracking power control (TTPC) was proposed in [10], and soft dropping power control (SDPC) was presented in [11]. With TTPC, the transmit power of each user is adjusted so that the SINR is maintained at or above a desired value. This power is an increasing function of the ratio of interference power to the channel gain experienced by the user. With SDPC, an increase in interference or a decrease in channel gain results in an increase in transmit power, so the target SINR is lowered. In [12], joint TTPC and SDPC power control (TSPC) schemes were proposed. With TSPC, two power control strategies are used. 
The first considers user target SINRs while minimizing the total transmit power while the second adjusts the target SINR according to the transmit power.

Game theoretic approaches to maximize the energy efficiency were employed for distributed power control in [1316]. The energy efficiency is expressed as a utility function which is the ratio of user throughput, defined as the number of information bits received with no error in a unit time, to transmit power. In [15], an efficient target SINR-oriented power control (TOPC) strategy was proposed. The user quality of service (QoS) is represented by the target SINRs, and the energy efficiency is given by a utility function. An energy-efficient power control game was formulated in [16] based on a pricing scheme, and a Pareto optimal solution was obtained. In [17], a prioritized and selective power allocation scheme was proposed. The goal is to satisfy all priority users and as many remaining users as possible. In [18], power allocation algorithms to minimize power consumption and outage probability were proposed assuming Rayleigh fading channels. Power allocation to maximize throughput and energy efficiency with an outage probability constraint was considered in [19].

With most power allocation schemes, only the channel path loss is considered, and the interference is assumed to be Gaussian distributed. The Rician distribution is commonly used to model fading when the wireless link between users has a line of sight (direct path) component and a diffused (Rayleigh) component [20, 21]. Obstacles, particularly in dense urban environments, can cause shadowing which affects the performance of a power allocation scheme [22, 23]. A log normal distribution is commonly used to model shadowing and so is employed here.

The relationship between outage probability and normalized SINR over Rician fading channels is considered in this paper. The outage probability is the probability that the user SINR falls below a target SINR. The target SINR is satisfied by allocating power so that users with an outage probability greater than a threshold are removed. The proposed method can be interpreted as an efficient approach to adjusting user transmit power levels.

The contributions of this paper are given below.

(1) Expressions for the outage probability over Rician fading channels are derived. Upper and lower bounds on the outage probability are obtained and shown to be tight. Unlike previous approaches, the outage probability is derived using the moment-generating function (MGF).

(2) A power allocation problem to minimize the power consumption with an outage probability threshold and SINR target is proposed. This threshold is determined so that users are satisfied with their target SINRs and the problem is solved iteratively.

(3) A power allocation problem to improve the energy efficiency with a target SINR constraint is proposed. This is a fractional programming problem and so is converted into a quadratic form using a parametric transformation.
(4) Two new power allocation schemes are presented which are shown to outperform existing schemes in terms of the number of satisfied users with the first scheme and power consumption and energy efficiency with the second scheme.

The remainder of this paper is organized as follows. The channel model is given in Section 2 and expressions for the outage probability and energy efficiency are presented. The power allocation problems considering normalized SINR and outage probability are given in Section 3. The performance of the proposed approaches is evaluated in Section 4, and finally, some concluding remarks are given in Section 5.

\section{System Model}

Radio channels have a significant impact on the performance of wireless communication systems and are affected by factors such as path loss, shadowing, and fading. Path loss characterizes the power decay due to the transmission distance, and shadowing represents the power absorption by obstacles. Consider a single cell with $n$ users and one base station (BS) located at the center as shown in Figure 1. The BS is equipped with a single antenna, and users are randomly distributed in the geographic area of the cell. Users share the same resources, so there is mutual interference due to concurrent transmissions.

Let the transmit power of user $i$ be $p_{i}$. Considering path loss and log normal shadowing, the channel gain can be written as [24-26]

$$
h_{i i}=\bar{g} d_{i i}^{-\alpha} 10^{\Xi / 10},
$$

where $\bar{g}=0.97$ is a constant, $d_{i i}$ is the distance between user $i$ and the BS, $\alpha$ is the path loss exponent, and $\Xi$ is a Gaussian random variable which represents the shadowing. A number of values for $\alpha$ have been proposed for different propagation environments, e.g., for urban environments $\alpha=4$ is often used.

The average received power for user $i$ is given by $[27,28]$

$$
\bar{p}_{i}=\mathbb{E}\left[h_{i i} u_{i i} p_{i}\right]=h_{i i} p_{i},
$$

where $u_{i i}$ denotes the fading component for user $i$ which is assumed to be a Rician distributed random variable with unit mean $\left(\bar{u}_{i i}=1\right)$ and $\mathbb{E}[\cdot]$ denotes expectation. The probability density function (pdf) of $u_{i i}$ is given by [29]

$$
f(u)=\left(\begin{array}{l}
\left(\frac{1+K}{\bar{u}}\right) \exp \left(-K-\frac{(1+K) u}{\bar{u}}\right) I_{0}\left(2 \sqrt{\frac{K(K+1) u}{\bar{u}}}\right), \quad \text { if } u \geq 0, \\
0, \quad \text { if } u<0,
\end{array}\right.
$$




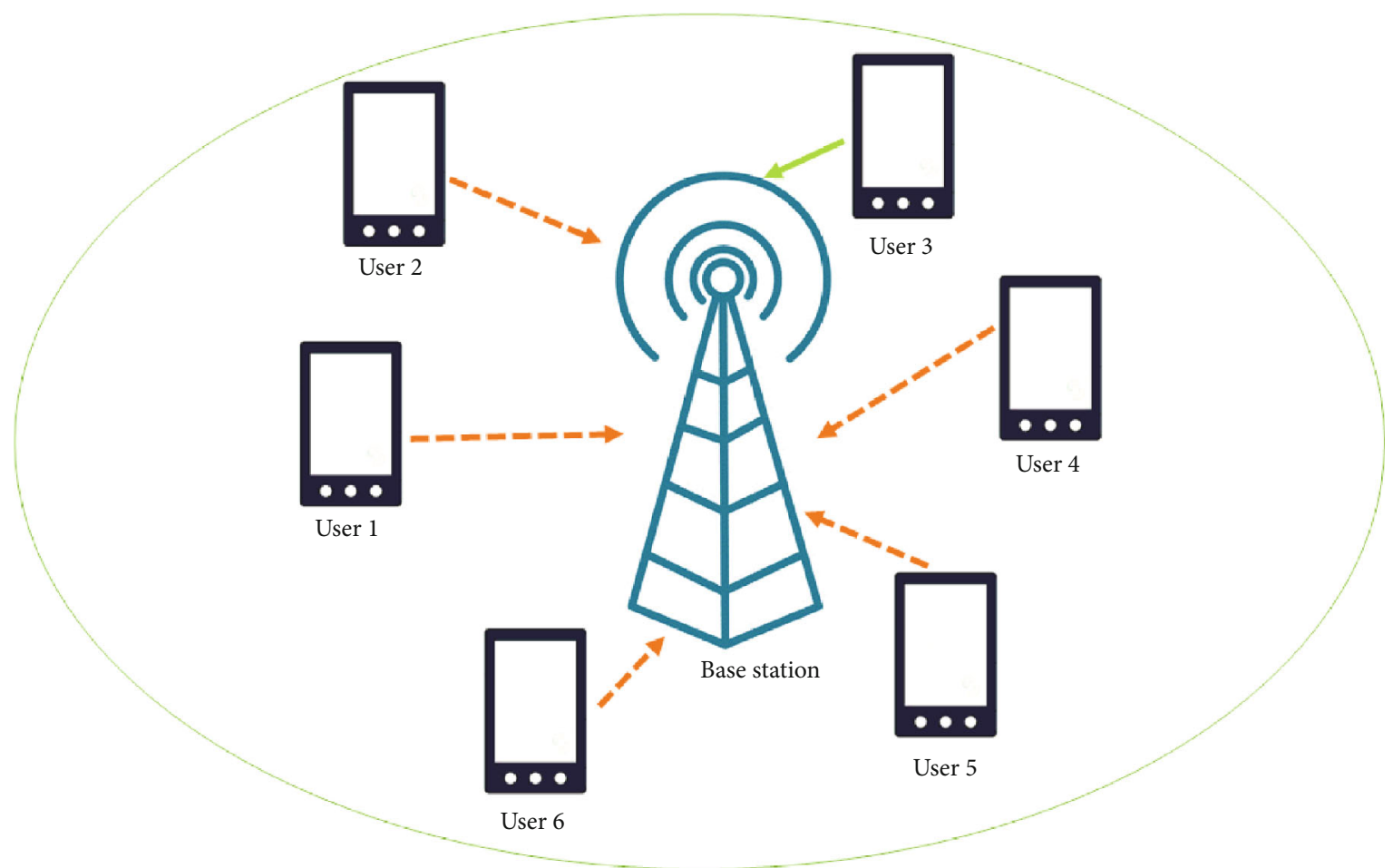

$\longrightarrow$ Desired users
$-\rightarrow$ Interfering users

Figure 1: The system model.

where $\bar{u}=\mathbb{E}[u], I_{0}(\cdot)$ is the modified Bessel function of the first kind with order zero, and $K \geq 0$ is the Rician fading parameter.

2.1. Throughput and Energy Efficiency. The SINR of user $i$ is given by

$$
\gamma_{i}=\frac{h_{i i} u_{i i} p_{i}}{\sum_{j \neq i} h_{i j} u_{i j} p_{j}+\sigma_{i}^{2}},
$$

where $\sigma_{i}^{2}$ denotes the additive white Gaussian noise (AWGN) at user $i$ and $u_{i j}$ and $h_{i j}$ are the associated Rician fading component and channel gain of the interference from user $j$ to user $i$, respectively.

The average SINR of user $i$ considering the interference from other users and noise is given by

$$
\bar{\gamma}_{i}=\frac{\mathbb{E}\left[h_{i i} u_{i i} p_{i}\right]}{\mathbb{E}\left[\sum_{j \neq i} h_{i j} u_{i j} p_{j}+\sigma_{i}^{2}\right]}=\frac{h_{i i} p_{i}}{\sum_{j \neq i} h_{i j} p_{j}+\sigma_{i}^{2}} .
$$

The normalized SINR of user $i$ is defined as

$$
\begin{aligned}
\bar{\Gamma}_{i} & =\frac{\bar{\gamma}_{i}}{\widehat{\gamma}_{i}}=\frac{h_{i i} p_{i}}{\widehat{\gamma}_{i}\left\{\sum_{j \neq i} h_{i j} p_{j}+\sigma_{i}^{2}\right\}} \\
& =\frac{p_{i}}{\widehat{\gamma}_{i}\left(\sum_{j \neq i}\left(h_{i j} / h_{i i}\right) p_{j}+\left(\sigma_{i}^{2} / h_{i i}\right)\right)} \\
& =\frac{p_{i}}{\widehat{\gamma}_{i}\left(\sum_{j \neq i} H_{i j} p_{j}+\bar{\sigma}_{i}\right)},
\end{aligned}
$$

where $\widehat{\gamma}_{i}$ is the target SINR, $H_{i j}=h_{i j} / h_{i i}$, and $\bar{\sigma}_{i}=\sigma_{i}^{2} / h_{i i}$. The corresponding throughput can be approximated as

$$
R_{i}=\log \left(1+\bar{\Gamma}_{i}\right)
$$
31]

The power consumption of user $i$ can be expressed as [30,

$$
P_{i}=\zeta p_{i}+p_{i c}
$$

where $\zeta$ denotes the reciprocal of the drain efficiency of the power amplifier, $\zeta \geq 0$, and $p_{i c}$ is the static part of the circuit 
power consumption that is independent of the radiated power and is due to functions such as signal processing. The energy efficiency of user $i$ can be expressed as

$$
Q_{i}=\frac{R_{i}}{P_{i}}
$$

so the system energy efficiency is

$$
q=\sum_{i=1}^{n} Q_{i}=\sum_{i=1}^{n} \frac{R_{i}}{P_{i}}
$$

2.2. Outage Probability. The outage probability is defined as the probability that the target SINR for a user is not achieved which for user $i$ can be expressed as

$$
O_{i}=\mathbb{P}\left(\gamma_{i} \leq \widehat{\gamma}_{i}\right)=\mathbb{P}\left(h_{i i} u_{i i} p_{i} \leq \widehat{\gamma}_{i}\left(\sum_{j \neq i} h_{i j} u_{i j} p_{j}+\sigma_{i}^{2}\right)\right)
$$

The outage probability considering the worst case channel for a user $K=0[32,33]$ can be written as [34-36]

$$
O_{i}=1-\exp \left(-\frac{\Lambda_{i}}{\bar{p}_{i}}\right) \prod_{j=1, j \neq i}^{n} \phi_{j}\left(\frac{\widehat{\gamma}_{i}}{\bar{p}_{i}}\right)
$$

where $\Lambda_{i}=\sigma_{i}^{2} \widehat{\gamma}_{i}, \bar{p}_{i}$ is the mean of the received power, and $\phi_{j}(\cdot)$ is the moment-generating function (MGF) of the interference to user $i$.

The MGF of the Rician distributed interference from user $j$ to user $i$ is

$$
\begin{gathered}
\phi_{j}\left(\frac{\left.\widehat{\gamma}_{i}\right)}{\bar{p}_{i}}\right) \\
=\int_{-\infty}^{\infty} \exp \left(-\frac{\widehat{\gamma}_{i}}{\bar{p}_{i}} p_{j}\right) f\left(p_{j}\right) d p_{j} \\
=\int_{0}^{\infty} \exp \left(-\frac{\widehat{\gamma}_{i}}{\bar{p}_{i}} p_{j}\right)\left(\frac{1+K}{\bar{p}_{j}}\right) \exp \left(-K-\frac{(1+K) p_{j}}{\bar{p}_{j}}\right) I_{0}\left(2 \sqrt{\frac{K(K+1) p_{j}}{\bar{p}_{j}}}\right) d p_{j} \\
=\frac{1+K}{1+K+\left(\widehat{\gamma}_{i} / \bar{p}_{i}\right) \bar{p}_{j}} \exp \left(\frac{-K\left(\widehat{\gamma}_{i} / \bar{p}_{i}\right) \bar{p}_{j}}{1+K+\left(\widehat{\gamma}_{i} / \bar{p}_{i}\right) \bar{p}_{j}}\right)
\end{gathered}
$$

where $\bar{p}_{j}$ is the mean of the interference to user $i$. From (12) and (13), the outage probability is

$$
\begin{aligned}
& O_{i} \quad=1-\exp \left(-\frac{\Lambda_{i}}{\bar{p}_{i}}\right) \prod_{j=1, j \neq i}^{n} \frac{1+K}{1+K+\left(\hat{\gamma}_{i} / \bar{p}_{i}\right) \bar{p}_{j}} \exp \left(\frac{-K\left(\widehat{\gamma}_{i} / \bar{p}_{i}\right) \bar{p}_{j}}{1+K+\left(\widehat{\gamma}_{i} / \bar{p}_{i}\right) \bar{p}_{j}}\right) \\
&=1-\exp \left(-\frac{\Lambda_{i}}{h_{i i} p_{i}}\right) \prod_{j=1, j \neq i}^{n} \frac{1+K}{1+K+\left(\widehat{\gamma}_{i} / h_{i i} p_{i}\right) h_{i j} p_{j}} \exp \left(\frac{-K\left(\widehat{\gamma}_{i} / h_{i i} p_{i}\right) h_{i j} p_{j}}{1+K+\left(\widehat{\gamma}_{i} / h_{i i} p_{i}\right) h_{i j} p_{j}}\right) .
\end{aligned}
$$

\section{Problem Formulation}

In this section, upper and lower bounds on the outage probability over Rician fading channels are derived. Then, a power allocation problem to minimize power consumption with outage probability and normalized SINR constraints is proposed, as well as a power allocation problem to maximize energy efficiency subject to a normalized SINR constraint.

3.1. Upper and Lower Bounds on the Outage Probability. Consider the following inequalities [37-39]

$$
e^{a} \prod_{i=1}^{n}\left(1+x_{i}\right) \geq 1+a+\sum_{i=1}^{n} x_{i}
$$

$$
\begin{gathered}
\sum_{i=1}^{n} \frac{x_{i}}{y_{i}} \geq \frac{\sum_{i=1}^{n} x_{i}}{\sum_{i=1}^{n} y_{i}}, \\
\frac{z}{w} \geq \frac{z-b}{w-b},
\end{gathered}
$$

where $x_{1}, x_{2}, \cdots, x_{n} \geq 0, y_{1}, y_{2}, \cdots, y_{n} \geq 0, a, b, n \geq 0$, and $z$ and $w$ are variables with $z<w$. Using these inequalities, a lower bound on the outage probability for user $i$ is obtained as

$$
O_{i} \geq 1-(1+K)^{n}\left[\exp (K)\left(1+n K+\frac{1}{\bar{\Gamma}_{i}}\right)\right]^{-1}
$$

The derivations are given in the appendix.

The outage probability in (14) can be rewritten as

$$
\begin{aligned}
O_{i}=1 & -\exp \left(-\frac{\bar{\sigma}_{i} \widehat{\gamma}_{i}}{p_{i}}\right)(1+K)^{n} \prod_{j=1, j \neq i}^{n}\left(1+K+\frac{\gamma \wedge_{i}}{h_{i i} p_{i}} h_{i j} p_{j}\right)^{-1} \\
& \cdot\left[\exp \left(\frac{\left(\gamma \wedge_{i} / h_{i i} p_{i}\right) h_{i j} p_{j}}{1+K+\left(\gamma \wedge_{i} / h_{i i} p_{i}\right) h_{i j} p_{j}}\right)\right]^{-K} .
\end{aligned}
$$


Using (17) and the following inequalities [40, 41]

$$
\begin{gathered}
\frac{y}{a+y} \leq \frac{y+b}{a+y}, \\
\frac{y}{1+y} \leq \ln (1+y), \\
e^{a} \prod_{i=1}^{n}\left(1+x_{i}\right) \leq e^{a} e^{\sum^{i=1}}\left(x_{i}\right),
\end{gathered}
$$

the upper bound on the outage probability is obtained as follows

$$
O_{i} \leq 1-\exp \left(-K \frac{\bar{\sigma}_{i} \widehat{\gamma}_{i}}{p_{i}}\right)(1+K)^{n}\left[\exp \left(n K+\frac{1}{\bar{\Gamma}_{i}}\right)\right]^{-(1+K)}
$$

The derivation is given in the appendix.

3.2. Power Allocation with Outage Probability and Normalized SINR Constraints. The power consumption problem subject to outage probability, normalized SINR, and transmit power constraints can be formulated as

$$
\begin{gathered}
\sum_{i=1}^{n} p_{i} \\
\text { minimize } \\
\text { subject to } \\
p_{\min } \leq p_{i} \leq p_{\max } \\
1-\exp \left(-\frac{\Lambda_{i}}{h_{i i} p_{i}}\right) \prod_{j=1, j \neq i}^{n} \frac{1+K}{1+K+\left(\widehat{\gamma}_{i} / h_{i i} p_{i}\right) h_{i j} p_{j}} \exp \left(\frac{-K\left(\widehat{\gamma}_{i} / h_{i i} p_{i}\right) h_{i j} p_{j}}{1+K+\left(\widehat{\gamma}_{i} / h_{i i} p_{i}\right) h_{i j} p_{j}}\right) \leq O_{\text {th }} \\
\bar{\Gamma}_{i} \geq 1,
\end{gathered}
$$

where $O_{\text {th }}$ is the outage probability threshold and $\bar{\Gamma}_{i}=\bar{\gamma}_{i} / \widehat{\gamma}_{i}$.

This is equivalent to

$$
\begin{gathered}
\sum_{i=1}^{n} p_{i} \\
\frac{p_{\min }}{p_{i}} \leq 1 \\
\frac{p_{i}}{p_{\max }} \leq 1 \\
\text { subject to } \frac{\hat{\gamma}_{i}}{\bar{\gamma}_{i}} \leq 1 \\
\frac{\exp \left(\Lambda_{i} / h_{i i} p_{i}\right)}{(1+K)^{n}}\left[\prod_{j=1, j \neq i}^{n}\left(1+K+\frac{\widehat{\gamma}_{i}}{h_{i i} p_{i}} h_{i j} p_{j}\right) \exp \left(\frac{K\left(\widehat{\gamma}_{i} / h_{i i} p_{i}\right) h_{i j} p_{j}}{1+K+\left(\widehat{\gamma}_{i} / h_{i i} p_{i}\right) h_{i j} p_{j}}\right)\right]\left(1-O_{\mathrm{th}}\right) \leq 1 .
\end{gathered}
$$

This is a geometric programming optimization problem with variables $p_{1}, p_{2}, \cdots, p_{k}$. Therefore, it can be solved using the interior point algorithm with convex optimization (CVX) geometry programming (GP) [42, 43]. Using the upper bound in (19) with the last constraint in (20) gives

$$
\begin{gathered}
1-\exp \left(-\frac{\Lambda_{i}}{h_{i i} p_{i}}\right) \prod_{j=1, j \neq i}^{n} \frac{1+K}{1+K+\left(\hat{\gamma}_{i} / h_{i i} p_{i}\right) h_{i j} p_{j}} \exp \left(\frac{-K\left(\widehat{\gamma}_{i} / h_{i} p_{i}\right) h_{i j} p_{j}}{1+K+\left(\widehat{\gamma}_{i} / h_{i i} p_{i}\right) h_{i j} p_{j}}\right) \\
\leq 1-\exp \left(-K \frac{\bar{\sigma}_{i} \widehat{\gamma}_{i}}{p_{i}}\right)(1+K)^{n}\left[\exp \left(n K+\frac{1}{\bar{\Gamma}_{i}}\right)\right]^{-(1+K)} \\
\leq O_{\text {th }} .
\end{gathered}
$$


This constraint is satisfied when the upper bound is less than $O_{\text {th }}$

$$
1-\exp \left(-K \frac{\bar{\sigma}_{i} \widehat{\gamma}_{i}}{p_{i}}\right)(1+K)^{n}\left[\exp \left(n K+\frac{1}{\bar{\Gamma}_{i}}\right)\right]^{-(1+K)} \leq O_{\text {th }}
$$

After some manipulation, the minimum transmit power that reduces the outage probability to at or below the threshold can be written as

$$
p_{i} \geq \frac{\widehat{\gamma}_{i}\left[K \bar{\sigma}_{i}+(1+K)\left(\sum_{j \neq i} H_{i j} p_{j}+\bar{\sigma}_{i}\right)\right]}{\ln \left[(1+K)^{n} /\left(1-O_{\text {th }}\right)\right]-(1+K) n K} .
$$

The power allocation problem then becomes

$$
\begin{gathered}
\sum_{i=1}^{n} p_{i} \\
\text { minimize } \\
\text { subject to } \quad \max \left\{\frac{\hat{\gamma}_{i}\left[K \bar{\sigma}_{i}+(1+K)\left(\sum_{j \neq i} H_{i j}+p_{j}+\bar{\sigma}_{i}\right)\right]}{\ln \left[(1+K)^{n} /\left(1-O_{\mathrm{th}}\right)\right]-(1+K) n K}, p_{\min }\right\} \leq p_{i} \leq p_{\max } \\
\bar{\gamma}_{i} \geq \widehat{\gamma}_{i} .
\end{gathered}
$$

This can be solved using the following power update function

$$
p_{i}(t+1)=\min \left\{\max \left\{\frac{\widehat{\gamma}_{i}\left[K \bar{\sigma}_{i}+(1+K)\left(\sum_{j \neq i} H_{i j} p_{j}(t)+\bar{\sigma}_{i}\right)\right]}{\ln \left[(1+K)^{n} /\left(1-O_{\mathrm{th}}\right)\right]-(1+K) n K}, \exp \left(\widehat{\gamma}_{i}(t)-\bar{\gamma}_{i}\right) p_{i}(t), p_{\min }\right\}, p_{\max }\right\} .
$$

A power allocation algorithm is obtained by defining the control parameter

$$
\kappa_{i}=\frac{1-O_{i}}{1-O_{\text {th }}} .
$$

The transmit power is increased if the outage probability is less than the threshold and decreased if greater than the threshold. Thus, if the outage probability of user $i$ is more than $O_{\text {th }}, \kappa$ is reduced resulting in a lower transmit power. The proposed power control algorithm to solve (20) based on (26) and (27) is given in Algorithm 1. The power allocation problem (20) is a concave function, but the transformed problem (25) which optimizes $p_{i}$ in (26) is a convex function. This problem can be solved iteratively to obtain the globally optimum solution of (20). Therefore, the number of iterations can be used as a measure of the complexity.

\subsection{Distributed Power Allocation for Energy Efficiency with a} Normalized SINR Constraint. The energy efficiency problem subject to normalized SINR and transmit power constraints is now presented. This problem can be formulated as

$$
\begin{array}{cc}
\text { maximize } & \sum_{i=1}^{n} Q_{i}=\sum_{i=1}^{n} \frac{R_{i}}{P_{i}} \\
\text { subject to } & p_{\min } \leq p_{i} \leq p_{\max } \\
& \bar{\Gamma}_{i} \geq 1,
\end{array}
$$

which is equivalent to

$$
\begin{array}{cl}
\text { maximize } \quad \sum_{i=1}^{n} Q_{i} & =\sum_{i=1}^{n} \frac{R_{i}}{P_{i}} \\
\text { subject to } \quad \frac{p_{\min }}{p_{i}} & \leq 1 \\
& \frac{p_{i}}{p_{\max }} \leq 1 \\
& \frac{\widehat{\gamma}_{i}}{\bar{\gamma}_{i}} \leq 1 .
\end{array}
$$

This is a geometric programming problem with variables $p_{1}, p_{2}, \cdots, p_{k}$. Therefore, it can be solved using the interior point method for GP problems [42, 43].

To obtain a subtractive form for the objective function, let

$$
V_{i}=v_{i} R_{i}-v_{i}^{2} P_{i}^{2}
$$

Taking the derivative of $V_{i}$ with respect to $v_{i}$ and setting it to 0 gives

$$
v_{i}=\frac{R_{i}}{2 P_{i}^{2}}
$$

Substituting $v_{i}$ in (30) gives

$$
V_{i}=v_{i} R_{i}-v_{i}^{2} P_{i}^{2}=\frac{R_{i}}{2 P_{i}^{2}} R_{i}-\left(\frac{R_{i}}{2 P_{i}^{2}}\right)^{2} P_{i}^{2}=\frac{R_{i}^{2}}{4 P_{i}^{2}} .
$$


Step 1. Initialize the iteration index $t=1$. User $i$ obtains the outage probability threshold $O_{\text {th }}$ from the BS, determines the average SINR $\bar{\gamma}_{i}$, and sets the target SINR $\widehat{\gamma}_{i}$.

Step 2. Users update their transmit power using

$p_{i}(t+1)=\min \left\{\max \left\{\kappa_{i}\left(\widehat{\gamma}_{i}\left[K \bar{\sigma}_{i}+(1+K)\left(\sum_{j \neq i} H_{i j} p_{j}(t)+\bar{\sigma}_{i}\right)\right] / \ln \left[(1+K)^{n} /\left(1-O_{\mathrm{th}}\right)\right]-(1+K) n K\right), \kappa_{i} \exp \left(\widehat{\gamma}_{i}(t)-\bar{\gamma}_{i}\right) p_{i}(t), p_{\min }\right\}\right.$, $\left.p_{\max }\right\}, i=1,2, \cdots, n$.

Step 3. Terminate when

$\max _{i}\left|p_{i}(t+1)-p_{i}(t)\right| \leq \varepsilon$,

otherwise, go to Step 2.

Algorithm 1: Power allocation algorithm with outage probability and normalized SINR constraints.

Since $R_{i} \geq 0$ and $P_{i} \geq 0$, maximizing $\sum_{i=1}^{n} Q_{i}=\sum_{i=1}^{n}\left(R_{i} / P_{i}\right)$ is equivalent to maximizing $\sum_{i=1}^{n} V_{i}^{2}=\sum_{i=1}^{n}\left(R_{i}^{2} / 4 P_{i}^{2}\right)$.

The power allocation problem (28) can be rewritten as

$$
\begin{array}{cl}
\operatorname{maximize} \quad \sum_{i=1}^{n} V_{i}= & \sum_{i=1}^{n}\left(v_{i} R_{i}-v_{i}^{2} P_{i}^{2}\right) \\
\text { subject to } \quad & \frac{p_{\min }}{p_{i}} \leq 1 \\
& \frac{p_{i}}{p_{\max }} \leq 1 \\
& \bar{\gamma}_{i} \geq \widehat{\gamma}_{i} .
\end{array}
$$

The system throughput $R_{i}$ is given by

$$
R_{i}=\log \left[1+\bar{\Gamma}_{i}\right]=\log \left[1+\frac{p_{i}}{\widehat{\gamma}_{i}\left(\sum_{j \neq i} H_{i j} p_{j}+\bar{\sigma}_{i}\right)}\right] \text {. }
$$

Let

$$
\Omega_{i}=1+\frac{p_{i}}{\widehat{\gamma}_{i}\left(\sum_{j \neq i} H_{i j} p_{j}+\bar{\sigma}_{i}\right)}=\frac{\widehat{\gamma}_{i}\left(\sum_{j \neq i} H_{i j} p_{j}+\bar{\sigma}_{i}\right)+p_{i}}{\widehat{\gamma}_{i}\left(\sum_{j \neq i} H_{i j} p_{j}+\bar{\sigma}_{i}\right)} .
$$

Substituting (8) and $R_{i}=\log \left(\Omega_{i}\right)$ in (31) gives

$$
v_{i}=\frac{R_{i}}{2 P_{i}^{2}}=\frac{\log \left(\Omega_{i}\right)}{2\left(\zeta p_{i}+p_{i c}\right)^{2}} .
$$

A subtractive form of $R_{i}$ can be obtained using $\Omega_{i}$ as follows

$$
\begin{gathered}
R_{i} \quad \log \left(\Omega_{i}\right)-\Omega_{i}+1+\frac{p_{i}}{\widehat{\gamma}_{i}\left(\sum_{j \neq i} H_{i j} p_{j}+\bar{\sigma}_{i}\right)} \\
=\log \left(\Omega_{i}\right)-\Omega_{i}+1+\frac{p_{i}}{\hat{\gamma}_{i}\left(\sum_{j \neq i} H_{i j} p_{j}+\bar{\sigma}_{i}\right)} \frac{\left.\widehat{\gamma}_{i}\left(\sum_{j \neq i} H_{i j} p_{j}+\bar{\sigma}_{i}\right)+p_{i j i} H_{i j} p_{j}+\bar{\sigma}_{i}\right)+p_{i}}{\widehat{\gamma}_{i}\left(\sum_{j \neq i} H_{i j} p_{j}+\bar{\sigma}_{i}\right)+p_{i}} \\
=\log \left(\Omega_{i}\right)-\Omega_{i}+1+\frac{\Omega_{i} p_{i}}{\widehat{c}_{i}}-\log \left(\Omega_{i}\right)-\Omega_{i}+1+l_{i} \sqrt{p_{i} \Omega_{i}}-l_{i}^{2}\left[p_{i}+\widehat{\gamma}_{i}\left(\sum_{j \neq i} H_{i j} p_{j}+\bar{\sigma}_{i}\right)\right],
\end{gathered}
$$

where $l_{i}$ is an additional variable. This variable can be determined by taking the derivative of $R_{i}$ with respect to $l_{i}$ and setting $\partial R_{i} / \partial l_{i}$ to 0 which gives

$$
l_{i}=\frac{\sqrt{p_{i} \Omega_{i}}}{2\left[p_{i}+\widehat{\gamma}_{i}\left(\sum_{j \neq i} H_{i j} p_{j}+\bar{\sigma}_{i}\right)\right]} .
$$

Using (8) and (37), the power allocation problem (33) can be reformulated as

maximize

$$
\sum_{i=1}^{n} V_{i}=\sum_{i=1}^{n} v_{i}\left\{\log \left(\Omega_{i}\right)-\Omega_{i}+1+l_{i} \sqrt{p_{i} \Omega_{i}-l_{i}^{2}\left[p_{i}+\widehat{\gamma}_{i}\left(\sum_{j \neq i} H_{i j} p_{j}+\bar{\sigma}_{i}\right)\right]}\right\}-\sum_{i=1}^{k} v_{i}^{2}\left(\zeta p_{i}+p_{i c}\right)^{2}
$$

subject to

$$
\begin{gathered}
\frac{p_{\min }}{p_{i}} \leq 1 \\
\frac{p_{i}}{p_{\max }} \leq 1 \\
\bar{\gamma}_{i} \geq \widehat{\gamma}_{i} .
\end{gathered}
$$


Step 1. Initialize the iteration index $t=1$. User $i$ determines the average SINR $\bar{\gamma}_{i}$ and sets the target $\operatorname{SINR} \widehat{\gamma}_{i}$.

Step 2. User $i$ determines $\Omega_{i}(t)$ using (35) and (6)

$\Omega_{i}(t)=1+\left(p_{i}(t) / \widehat{\gamma}_{i}\left[\sum_{j \neq i} H_{i j} p_{j}(t)+\bar{\sigma}_{i}\right]\right)=1+\left(\bar{\gamma}_{i}(t) / \widehat{\gamma}_{i}\right), i=1,2, \cdots, n$.

Step 3. User $i$ determines $v_{i}(t)$ using (36)

$v_{i}(t)=\log \left(\Omega_{i}(t)\right) / 2\left(\zeta p_{i}(t)+p_{i c}\right)^{2}, i=1,2, \cdots, n$.

Step 4. User $i$ determines $l_{i}(t)$ using (38) and (6)

$l_{i}(t)=\sqrt{p_{i}(t) \Omega_{i}} / 2\left[p_{i}(t)+\widehat{\gamma}_{i}\left(\sum_{j \neq i} H_{i j} p_{j}(t)+\bar{\sigma}_{i}\right)\right]=\sqrt{p_{i}(t) \Omega_{i}} / 2 p_{i}(t)\left[1+\left(\widehat{\gamma}_{i} /\left(\bar{\gamma}_{i}(t)\right)\right)\right], i=1,2, \cdots, n$.

Step 5. User $i$ updates the transmit power using (40) and (41)

$p_{i}(t+1)=\min \left\{\max \left(l_{i}(t) \Omega_{i} / 2\left[v_{i}(t) \zeta+l_{i}(t)^{2}\left(1+\widehat{\gamma}_{i} \sum_{j \neq i} H_{i j}\right)\right],\left(\sum_{j \neq i} H_{i j} p_{j}(t)+\bar{\sigma}_{i}\right) \widehat{\gamma}_{i}\right), p_{\min }, p_{\max }\right\}, i=1,2, \cdots, n$.

Step 6. Terminate if

$\max _{i}\left|p_{i}(t+1)-p_{i}(t)\right| \leq \varepsilon$,

otherwise, go to Step 2.

Algorithm 2: Distributed power allocation for energy efficiency with a normalized SINR constraint.

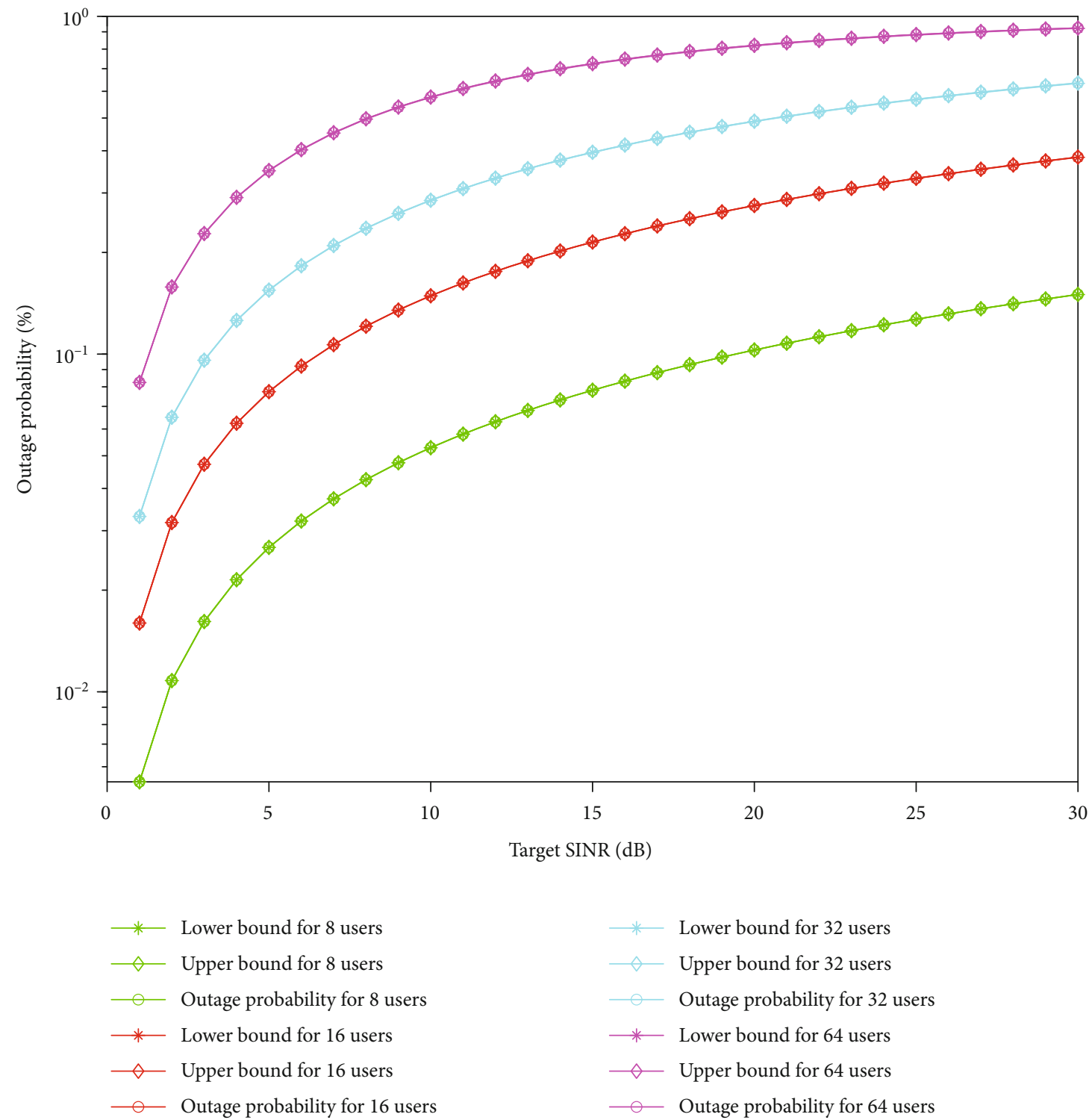

FIGURE 2: Upper and lower bounds on the outage probability with different numbers of users. 


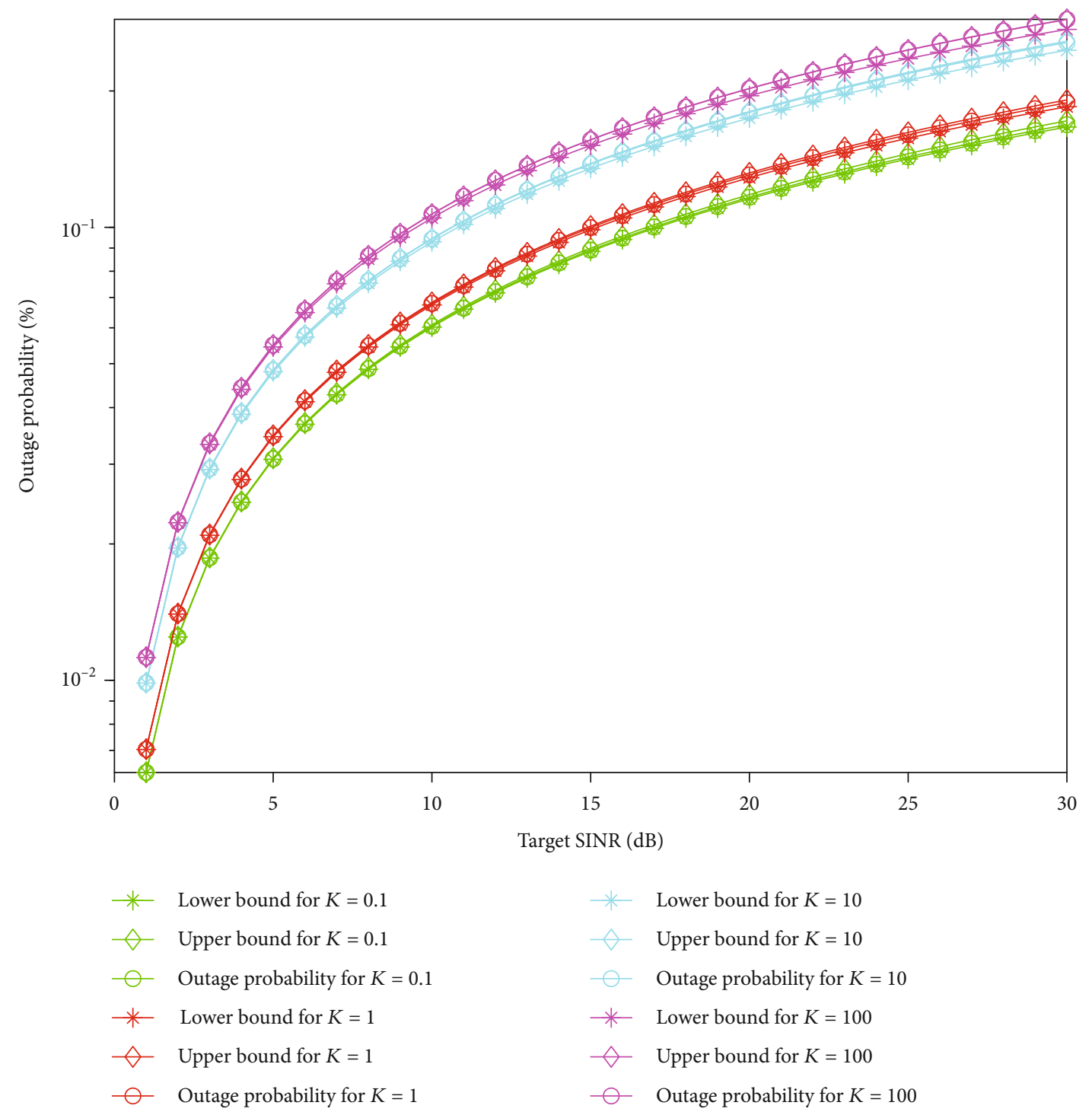

FIGURE 3: Upper and lower bounds on the outage probability with different values of $K$.

Taking the derivative of $V_{i}$ with respect to $p_{i}$ and setting $\partial V_{i} / \partial p_{i}$ to 0 gives

$$
p_{i}=\frac{l_{i} \Omega_{i}}{2\left[v_{i} \zeta+l_{i}^{2}\left(1+\widehat{\gamma}_{i} \sum_{j \neq i} H_{i j}\right)\right]} .
$$

The minimum transmit power to attain the target SINR is obtained from the last constraint in (37) as

$$
p_{i} \geq\left(\sum_{j \neq i} H_{i j} p_{j}+\bar{\sigma}_{i}\right) \widehat{\gamma}_{i} .
$$

The proposed distributed power control algorithm to solve (39) based on (35), (36), (38), (40), and (41) is given in Algorithm 2. Since (39) is a maximization problem, $\Omega_{i}$ in (35), $v_{i}$ in (36), and $l_{i}$ in (38) increase or at least do not decease each iteration. Therefore, Algorithm 2 will converge by alternatively updating $\Omega_{i}, v_{i}$, and $l_{i}$ and then solving for $p_{i}$ in (40). In particular, the power allocation problem (29) is a concave fractional programming problem, but the transformed problem (39) which optimizes $p_{i}$ in (40) for fixed $\Omega_{i}$, $v_{i}$, and $l_{i}$ is convex. Thus, it can be solved iteratively to obtain the globally optimum solution of (29).

\section{Numerical Results}

In this section, the performance of the proposed power allocation schemes is evaluated using Monte Carlo simulation for different numbers of users and values of $K$. The path loss exponent in (1) is set to $\alpha=4$ which corresponds to typical urban and suburban environments [44-46]. The stopping criterion for the algorithms is set to $\varepsilon=10^{-20}$. The noise power is $\sigma^{2}=0.01 \mathrm{~W}$, the initial power level for all users is set to $0.01 \mathrm{~W}$, and the maximum transmit power is $p_{\max }=2$ W. The drain efficiency of the power amplifier is $\zeta=0.38$ 


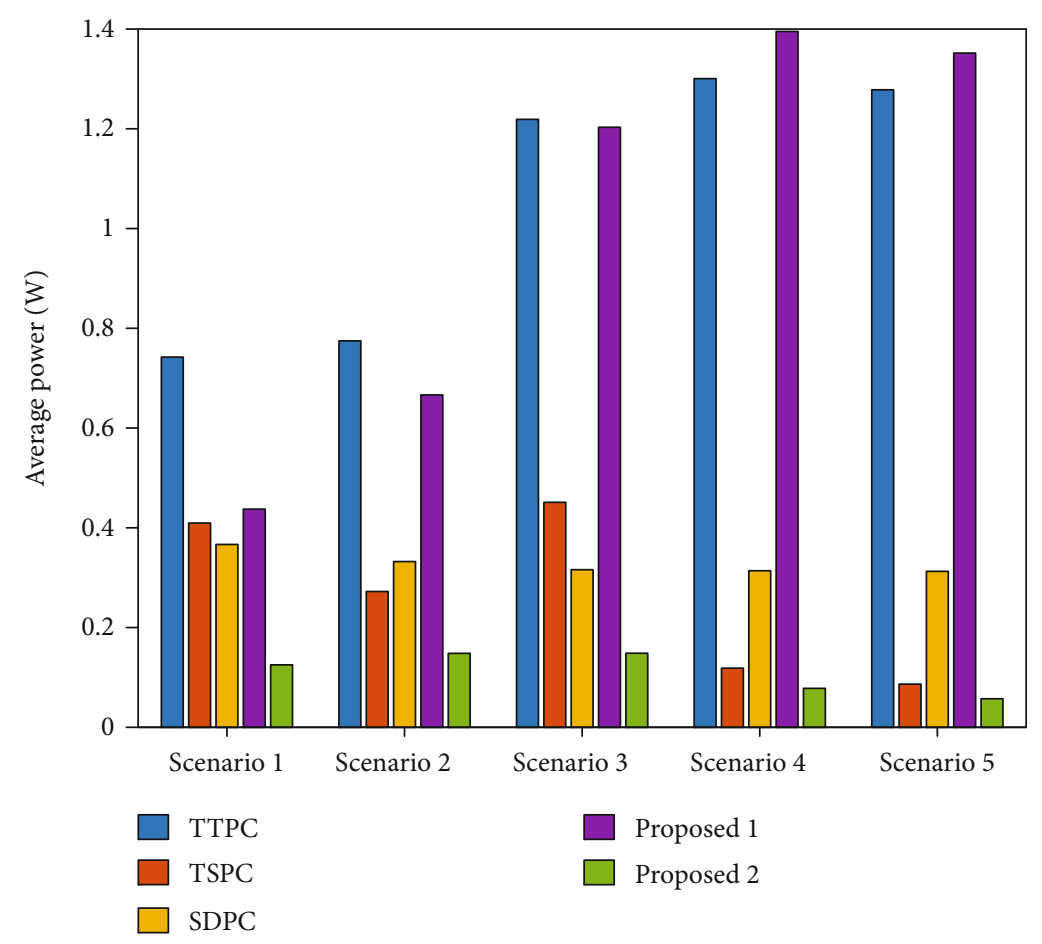

FIgURE 4: The average power for five scenarios with five power allocation schemes.

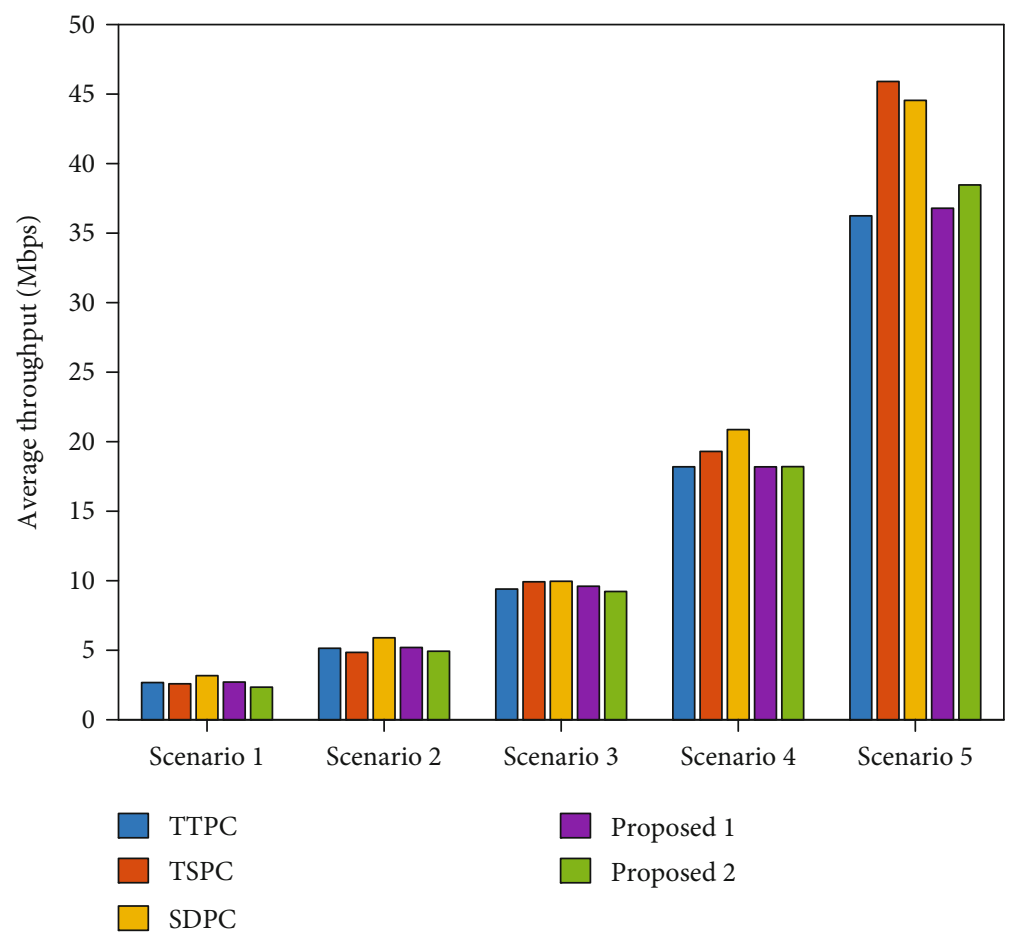

FIgURE 5: The average throughput for five scenarios with five power allocation schemes.

[47], and the static circuit power consumption is $p_{i c}=0.01 \mathrm{~W}$ [48].

The performance of the proposed power allocation schemes is compared with that of the target SINR tracking power control (TTPC), soft dropping power control (SDPC), and joint TTPC and SDPC power control (TSPC) schemes.
Power consumption, throughput, energy efficiency, outage probability, and number of unsatisfied users are the comparison criteria. The users are uniformly distributed in the geographic areas of the cells. In the first and second scenarios, there is a femtocell with 4 and 8 users, respectively. The BS is located at the center of a square geographic area with 


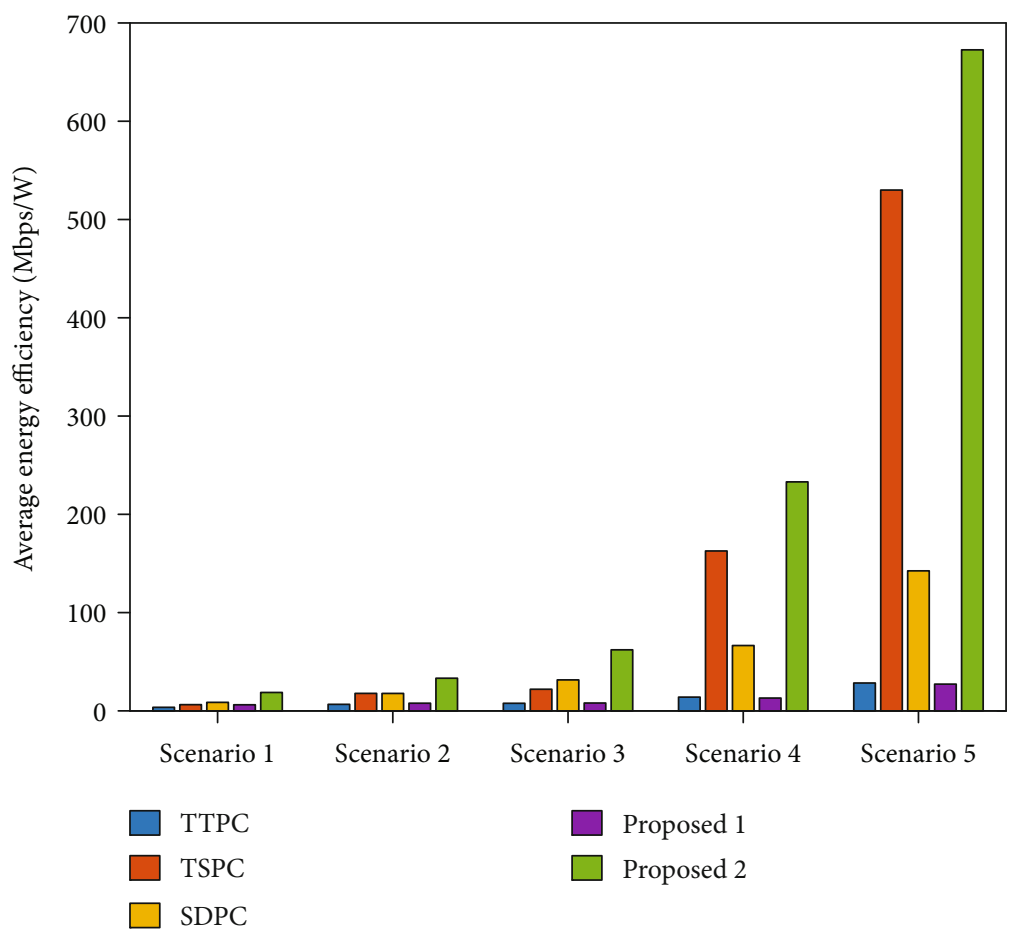

FIGURE 6: The average energy efficiency for five scenarios with five power allocation schemes.

dimensions $15 \mathrm{~m} \times 15 \mathrm{~m}$. In the third and fourth scenarios, there is a picocell with 16 and 32 users, respectively. The BS is located at the center of a square geographic area with dimensions $200 \mathrm{~m} \times 200 \mathrm{~m}$. In the fifth scenario, there is a macrocell with 64 users. The BS is located at the center of a square geographic area with dimensions $1000 \mathrm{~m} \times 1000 \mathrm{~m}$. The target SINRs were chosen randomly in the range [ $0.1,0.8] \mathrm{dB}$. The results are obtained for 1000 trials for each number of users with the user locations changed each trial.

Figure 2 presents the exact outage probability from (14) and the corresponding lower bound from (16) and upper bound from (19) with 8, 16, 32, and 64 users versus the target SINR for $K=50$. This shows that the upper and lower bounds are tight. The maximum approximation error using the lower bound is $0.42 \%$, and the maximum error using the upper bound is $0.04 \%$, which is slightly better than the lower bound. These were obtained with 64 users and an SINR of $30 \mathrm{~dB}$. Therefore, these bounds are suitable to estimate the outage probability in cellular networks. The exact outage probability for 8 users varies from $0.01 \%$ to $0.24 \%$, for 16 users from $0.02 \%$ to $0.37 \%$, for 32 users from $0.03 \%$ to $0.58 \%$, and for 64 users from $0.07 \%$ to $0.88 \%$. As expected, the outage probability is higher when the user density is higher.

Figure 3 presents the exact outage probability and the corresponding upper and lower bounds for different values of $K$ versus the target SINR. This shows that the upper bound is a better approximation than the lower bound. The maximum approximation errors using the lower bound for $K=$ $0.1,1,10$, and 100 are $0.11 \%, 0.28 \%, 0.59 \%$, and $0.89 \%$, respectively, while the corresponding errors using the upper bound for $K=0.1,1,10$, and 100 are $0.15 \%, 0.26 \%, 0.05 \%$, and $0.06 \%$, respectively. Note that the outage probability increases with $K$ as the interference is greater.
Figure 4 presents the average power for the TTPC, TSPC, SDPC, and proposed power allocation schemes for the five scenarios. This shows that the second proposed scheme has the lowest average power. In the first scenario, the average power for this scheme is $0.15 \mathrm{~W}$. This is followed by SDPC, TSPC, and the first proposed scheme with average power $0.37 \mathrm{~W}, 0.41 \mathrm{~W}$, and $0.44 \mathrm{~W}$, respectively. The performance of TTPC is the worst with an average power of $0.74 \mathrm{~W}$. In the second and third scenarios, the average power with the second proposed scheme is less than $0.14 \mathrm{~W}$, which is much better than TTPC, SDPC, TSPC, and the first proposed scheme. The average power for TTPC is the highest with $1.21 \mathrm{~W}$ for the second scenario and $1.30 \mathrm{~W}$ for the third scenario. The average power for the second proposed scheme is $0.08 \mathrm{~W}$ for the fourth scenario and $0.06 \mathrm{~W}$ for the fifth scenario, which are the lowest. This is followed by TSPC, SDPC, TTPC, and the first proposed scheme.

Figure 5 presents the average throughput from (7) for the TTPC, TSPC, SDPC, and proposed power allocation schemes for the five scenarios. This shows that the average throughput increases from scenario 1 to scenario 5 . The average throughput for SDPC is slightly higher than the others in the first four scenarios. For scenario 5, the average throughput for TSPC is $45.9 \mathrm{Mbps}$, which is the highest. This is followed closely by SDPC with an average throughput of $44.6 \mathrm{Mbps}$ and then the second proposed scheme with $38.6 \mathrm{Mbps}$. The average throughput for TTPC and the first proposed scheme is $36.2 \mathrm{Mbps}$ and $36.8 \mathrm{Mbps}$, respectively.

Figure 6 presents the average energy efficiency from (10) for the TTPC, TSPC, SDPC, and proposed power allocation schemes for the five scenarios. This shows that the second proposed scheme provides the highest energy efficiency. The average energy efficiency is $18.7 \mathrm{Mbps} / \mathrm{W}$ for the first 


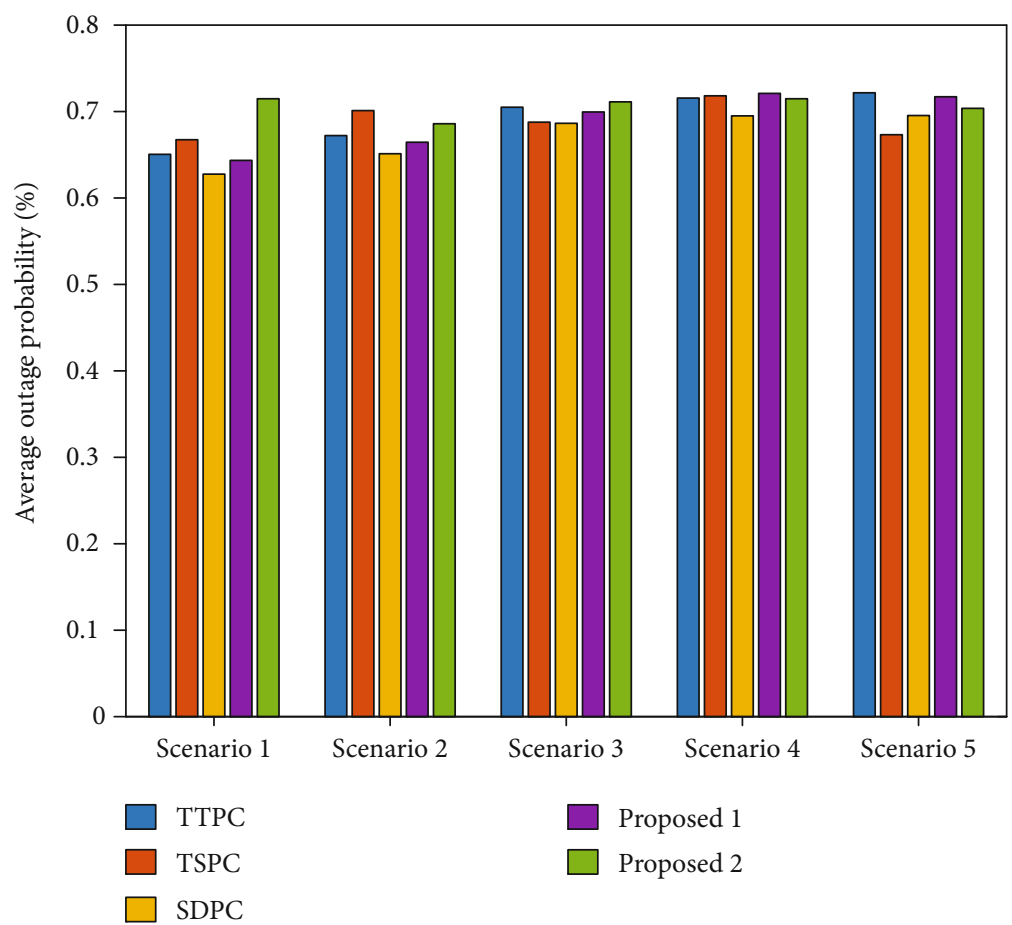

FIGURE 7: The average outage probability for five scenarios with five power allocation schemes.

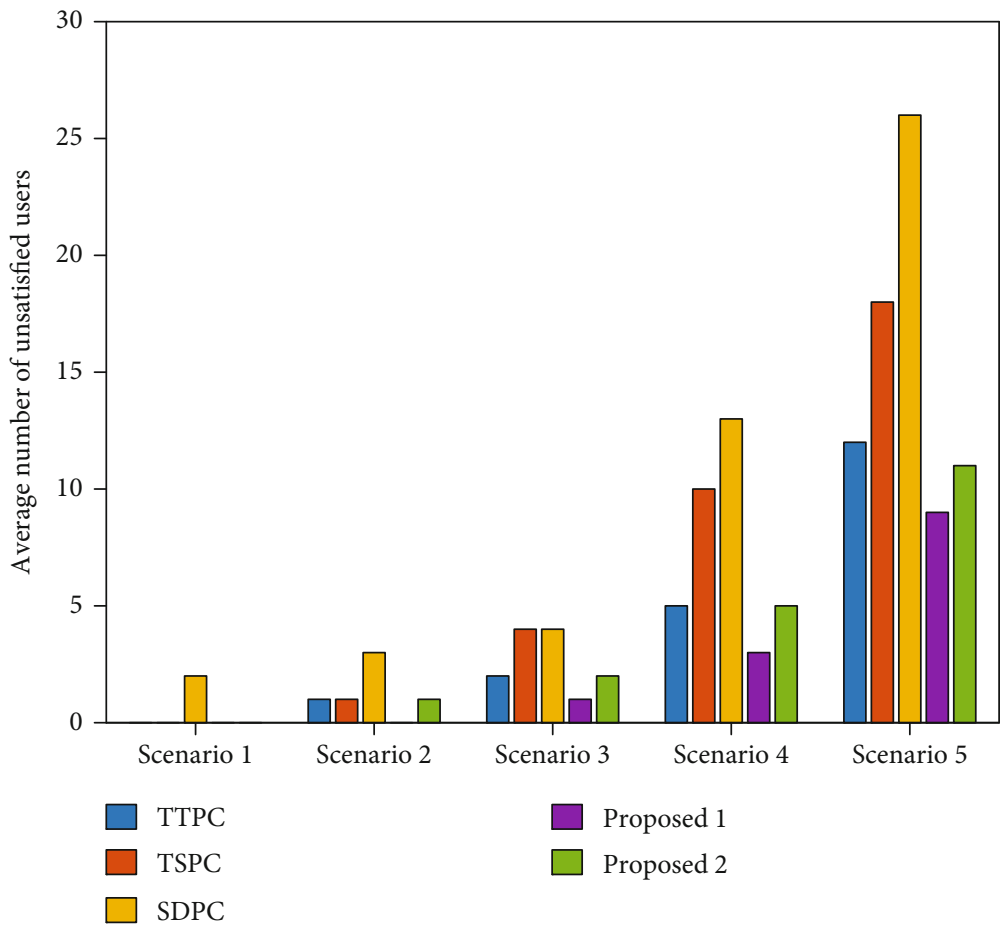

FIgURE 8: The average number of unsatisfied users for five scenarios with five power allocation schemes.

scenario, $33.2 \mathrm{Mbps} / \mathrm{W}$ for the second scenario, $62.1 \mathrm{Mbps} / \mathrm{W}$ for the third scenario, $233.0 \mathrm{Mbps} / \mathrm{W}$ for the fourth scenario, and $672.6 \mathrm{Mbps} / \mathrm{W}$ for the fifth scenario. This is followed by TSPC and then SDPC. TTPC and the first proposed scheme have the lowest energy efficiency.
Figure 7 presents the average outage probability for the TTPC, TSPC, SDPC, and proposed power allocation schemes for the five scenarios. This shows that the average outage probability for all five schemes is quite similar. Figure 8 presents the average number of unsatisfied users 
for the TTPC, TSPC, SDPC, and proposed power allocation schemes for the five scenarios. The average number of unsatisfied users is the lowest with the first proposed scheme, followed by the second proposed scheme, TTPC, and TSPC. The average number of unsatisfied users for SDPC is $2,3,4,13$, and 26 , which is the highest for all scenarios.

All of the algorithms considered are iterative, so the number of iterations is used as a measure of complexity. In all cases, four iterations were required for convergence so the algorithms have similar complexity. From the results obtained, TTPC provides the lowest average energy efficiency and this decreases as the number of users increases. This is because the goal of TTPC is to maximize the transmit power according to the channel conditions. TSPC and SDPC have the highest number of unsatisfied users since the goal of TSPC and SDPC is to maximize the throughput. Thus, users increase their transmit powers which cause more users to not be satisfied with their target SINRs. Algorithm 1 results in fewer unsatisfied users because the power update function incorporates both the normalized SINR and outage probability. Algorithm 2 provides good energy efficiency because the power update function incorporates both the channel conditions and throughput over power ratio.

\section{Conclusion}

In this paper, two power allocation schemes for throughput and energy efficiency in cellular networks were proposed. The goal is to reduce the power consumption and improve the energy efficiency while ensuring the user outage probability is below a threshold. Performance results were presented which show that the proposed schemes provide a higher energy efficiency than the target SINR tracking power control (TTPC), soft dropping power control (SDPC), and joint TTPC and SDPC power control (TSPC) schemes. The first proposed scheme had the lowest number of unsatisfied users for all scenarios, followed by the second proposed scheme and TTPC for all scenarios except scenario 2. The second proposed scheme had the highest average power and energy efficiency for all scenarios. The first proposed scheme and TTPC required the highest average power and so have the lowest energy efficiency. The average outage probability and throughput were similar for all five schemes.

\section{Appendix}

\section{Derivation of (16) and (19)}

A lower bound on the outage probability in (16) for user $i$ is obtained as follows.

$$
\begin{aligned}
& O_{i} \quad=1-\exp \left(-\frac{\Lambda_{i}}{h_{i i} p_{i}}\right) \prod_{j=1, j \neq i}^{n} \frac{1+K}{1+K+\left(\widehat{\gamma}_{i} / h_{i i} p_{i}\right) h_{i j} p_{j}} \exp \left(\frac{-K\left(\widehat{\gamma}_{i} / h_{i i} p_{i}\right) h_{i j} p_{j}}{1+K+\left(\widehat{\gamma}_{i} / h_{i i} p_{i}\right) h_{i j} p_{j}}\right) \\
& \stackrel{(15 \mathrm{a})}{\geq} 1-(1+K)^{n}\left[1+\frac{\bar{\sigma}_{i} \gamma \wedge_{i}}{p_{i}}+\sum_{j=1, j \neq i}^{n}\left(K+\frac{\gamma \wedge_{i} H_{i j} p_{j}}{p_{i}}\right)\right]^{-1} \prod_{j=1, j \neq i}^{n} \exp \left(\frac{-K\left(\widehat{\gamma}_{i} / h_{i i} p_{i}\right) h_{i j} p_{j}}{1+K+\left(\widehat{\gamma}_{i} / h_{i i} p_{i}\right) h_{i j} p_{j}}\right) \\
& =1-(1+K)^{n}\left(1+\frac{\bar{\sigma}_{i} \gamma \wedge_{i}}{p_{i}}+n K+\sum_{j=1, j \neq i}^{n} \frac{\gamma \wedge_{i} H_{i j} p_{j}}{p_{i}}\right)^{-1} \prod_{j=1, j \neq i}^{n} \exp \left(\frac{-K\left(\widehat{\gamma}_{i} / h_{i i} p_{i}\right) h_{i j} p_{j}}{1+K+\left(\widehat{\gamma}_{i} / h_{i i} p_{i}\right) h_{i j} p_{j}}\right) \\
& =1-(1+K)^{n}\left(1+\frac{\bar{\sigma}_{i} \gamma \wedge_{i}}{p_{i}}+n K+\sum_{j=1, j \neq i}^{n} \frac{\gamma \wedge_{i} H_{i j} p_{j}}{p_{i}}\right)^{-1} \exp \left(\sum_{j=1, j \neq i}^{n} \frac{-K\left(\widehat{\gamma}_{i} / h_{i i} p_{i}\right) h_{i j} p_{j}}{1+K+\left(\widehat{\gamma}_{i} / h_{i i} p_{i}\right) h_{i j} p_{j}}\right) \\
& \stackrel{(15 b)}{\geq}(1+K)^{n}\left(1+\frac{\bar{\sigma}_{i} \gamma \wedge_{i}}{p_{i}}+n K+\sum_{j=1, j \neq i}^{n} \frac{\gamma \wedge_{i} H_{i j} p_{j}}{p_{i}}\right)^{-1} \exp \left[\frac{-\sum_{j=1, j \neq i}^{n} K\left(\widehat{\gamma}_{i} / h_{i i} p_{i}\right) h_{i j} p_{j}}{\sum_{j=1, j \neq i}^{n}\left(1+K+\left(\widehat{\gamma}_{i} / h_{i i} p_{i}\right) h_{i j} p_{j}\right)}\right] \\
& =1-(1+K)^{n}\left(1+\frac{\bar{\sigma}_{i} \gamma \wedge_{i}}{p_{i}}+n K+\sum_{j=1, j \neq i}^{n} \frac{\gamma \wedge_{i} H_{i j} p_{j}}{p_{i}}\right)^{-1} \exp \left[\frac{-K \sum_{j=1, j \neq i}^{n}\left(\widehat{\gamma}_{i} / h_{i i} p_{i}\right) h_{i j} p_{j}}{n+n K+\sum_{j=1, j \neq i}^{n}\left(\left(\widehat{\gamma}_{i} / h_{i i} p_{i}\right) h_{i j} p_{j}\right)}\right] \\
& \stackrel{(15 c)}{\geq}(1+K)^{n}\left[1+\frac{\bar{\sigma}_{i} \gamma \wedge_{i}}{p_{i}}+n K+\sum_{j=1, j \neq i}^{n} \frac{\gamma \wedge_{i} H_{i j} p_{j}}{p_{i}}\right]^{-1} \exp \left[\frac{-K \sum_{j=1, j \neq i}^{n}\left(\widehat{\gamma}_{i} / h_{i i} p_{i}\right) h_{i j} p_{j}-n K}{n+n K+\sum_{j=1, j \neq i}^{n}\left(\left(\widehat{\gamma}_{i} / h_{i i} p_{i}\right) h_{i j} p_{j}\right)-n K}\right] \\
& =1-(1+K)^{n}\left(1+\frac{\bar{\sigma}_{i} \gamma \wedge_{i}}{p_{i}}+n K+\sum_{j=1, j \neq i}^{n} \frac{\gamma \wedge_{i} H_{i j} p_{j}}{p_{i}}\right)^{-1} \exp \left[\frac{-K\left(\sum_{j=1, j \neq i}^{n}\left(\widehat{\gamma}_{i} / h_{i i} p_{i}\right) h_{i j} p_{j}+n\right)}{n+\sum_{j=1, j \neq i}^{n}\left(\left(\widehat{\gamma}_{i} / h_{i i} p_{i}\right) h_{i j} p_{j}\right)}\right] \\
& =1-(1+K)^{n}\left(1+\frac{\bar{\sigma}_{i} \gamma \wedge_{i}}{p_{i}}+n K+\sum_{j=1, j \neq i}^{n} \frac{\gamma \wedge_{i} H_{i j} p_{j}}{p_{i}}\right)^{-1} \exp (-K) \\
& =1-(1+K)^{n}\left\{\exp (K)\left[1+n K+\frac{\gamma \wedge_{i}}{p_{i}}\left(\bar{\sigma}_{i}+\sum_{j=1, j \neq i}^{n} H_{i j} p_{j}\right)\right]\right\}^{-1} \\
& =1-(1+K)^{n}\left[\exp (K)\left(1+n K+\frac{1}{\bar{\Gamma}_{i}}\right)\right]^{-1} \text {. }
\end{aligned}
$$


The upper bound on the outage probability in (19) for user $i$ is obtained as follows.

$$
\begin{aligned}
& O_{i} \quad=1-\exp \left(-\frac{\bar{\sigma}_{i} \widehat{\gamma}_{i}}{p_{i}}\right)(1+K)^{n} \prod_{j=1, j \neq i}^{n}\left(1+K+\frac{\gamma \wedge_{i}}{h_{i i} p_{i}} h_{i j} p_{j}\right)^{-1}\left[\exp \left(\frac{\left(\gamma \wedge_{i} / h_{i i} p_{i}\right) h_{i j} p_{j}}{1+K+\left(\gamma \wedge_{i} / h_{i i} p_{i}\right) h_{i j} p_{j}}\right)\right]^{-K} \\
& \stackrel{(18 \mathrm{a})}{\leq} 1-\exp \left(-\frac{\bar{\sigma}_{i} \widehat{\gamma}_{i}}{p_{i}}\right)(1+K)^{n} \prod_{j=1, j \neq i}^{n}\left(1+K+\frac{\gamma \wedge_{i}}{h_{i i} p_{i}} h_{i j} p_{j}\right)^{-1}\left[\exp \left(\frac{\left(\gamma \wedge_{i} / h_{i i} p_{i}\right) h_{i j} p_{j}+K}{1+K+\left(\gamma \wedge_{i} / h_{i i} p_{i}\right) h_{i j} p_{j}}\right)\right]^{-K} \\
& \stackrel{(18 \mathrm{~b})}{\leq} 1-\exp \left(-\frac{\bar{\sigma}_{i} \widehat{\gamma}_{i}}{p_{i}}\right)(1+K)^{n} \prod_{j=1, j \neq i}^{n}\left(1+K+\frac{\gamma \wedge_{i}}{h_{i i} p_{i}} h_{i j} p_{j}\right)^{-1}\left\{\exp \left[\ln \left(1+K+\frac{\gamma \wedge_{i}}{h_{i i} p_{i}} h_{i j} p_{j}\right)\right]\right\}^{-K} \\
& =1-\exp \left(-\frac{\bar{\sigma}_{i} \widehat{\gamma}_{i}}{p_{i}}\right)(1+K)^{n} \prod_{j=1, j \neq i}^{n}\left(1+K+\frac{\gamma \wedge_{i}}{h_{i i} p_{i}} h_{i j} p_{j}\right)^{-1}\left(1+K+\frac{\gamma \wedge_{i}}{h_{i i} p_{i}} h_{i j} p_{j}\right)^{-K} \\
& =1-\exp \left(-\frac{\bar{\sigma}_{i} \widehat{\gamma}_{i}}{p_{i}}\right)(1+K)^{n} \prod_{j=1, j \neq i}^{n}\left(1+K+\frac{\gamma \wedge_{i}}{h_{i i} p_{i}} h_{i j} p_{j}\right)^{-(1+K)} \\
& \stackrel{(18 \mathrm{c})}{\leq} 1-\exp \left(-\frac{\bar{\sigma}_{i} \widehat{\gamma}_{i}}{p_{i}}\right)(1+K)^{n}\left\{\exp \left[\sum_{j=1, j \neq i}^{n}\left(K+\frac{\gamma \wedge_{i}}{h_{i i} p_{i}} h_{i j} p_{j}\right)\right]\right\}^{-(1+K)} \\
& =1-\exp \left(-\frac{\bar{\sigma}_{i} \widehat{\gamma}_{i}}{p_{i}}\right)(1+K)^{n}\left\{\exp \left[\sum_{j=1, j \neq i}^{n}\left(K+\frac{\gamma \wedge_{i}}{h_{i i} p_{i}} h_{i j} p_{j}\right)\right]\right\}^{-(1+K)} \frac{\left[\exp \left(\bar{\sigma}_{i} \gamma \wedge_{i} / p_{i}\right)\right]^{-(1+K)}}{\left[\exp \left(\bar{\sigma}_{i} \gamma \wedge_{i} / p_{i}\right)\right]^{-(1+K)}} \\
& =1-\exp \left(-K \frac{\bar{\sigma}_{i} \widehat{\gamma}_{i}}{p_{i}}\right)(1+K)^{n}\left\{\exp \left[\frac{\bar{\sigma}_{i} \gamma \wedge_{i}}{p_{i}}+\sum_{j=1, j \neq i}^{n}\left(K+\frac{\gamma \wedge_{i}}{h_{i i} p_{i}} h_{i j} p_{j}\right)\right]\right\}^{-(1+K)} \\
& =1-\exp \left(-K \frac{\bar{\sigma}_{i} \widehat{\gamma}_{i}}{p_{i}}\right)(1+K)^{n}\left\{\exp \left[n K+\frac{\gamma \wedge_{i}}{p_{i}}\left(\bar{\sigma}_{i}+\sum_{j=1, j \neq i}^{n} H_{i j} p_{j}\right)\right]\right\}^{-(1+K)} \\
& =1-\exp \left(-K \frac{\bar{\sigma}_{i} \widehat{\gamma}_{i}}{p_{i}}\right)(1+K)^{n}\left[\exp \left(n K+\frac{1}{\bar{\Gamma}_{i}}\right)\right]^{-(1+K)} \text {. }
\end{aligned}
$$

\section{Data Availability}

No data were used to support this study.

\section{Conflicts of Interest}

The authors declare no conflict of interest regarding this publication.

\section{References}

[1] Y. Liu, Y. Yang, P. Han, Z. Shao, and C. Li, "Virtual network embedding in fiber-wireless access networks for resourceefficient IoT service provisioning," IEEE Access, vol. 7, pp. 65506-65517, 2019.

[2] R. Boussada, B. Hamdane, M. E. Elhdhili, and L. A. Saidane, "Privacy-preserving aware data transmission for IoT-based ehealth," Computer Networks, vol. 162, article 106866, 2019.
[3] A. Rachedi, M. H. Rehmani, S. Cherkaoui, and J. J. P. C. Rodrigues, "IEEE access special section editorial: the plethora of research in Internet of things (IoT)," IEEE Access, vol. 4, pp. 9575-9579, 2016.

[4] Z. Wang, J. Wang, Y. Zhang, and D. Niyato, "Strategic access and pricing in Internet of things (IoT) service with energy harvesting," IEEE Access, vol. 7, pp. 34655-34674, 2019.

[5] A. Musaddiq, Y. B. Zikria, O. Hahm, H. Yu, A. K. Bashir, and S. W. Kim, "A survey on resource management in IoT operating systems," IEEE Access, vol. 6, pp. 8459-8482, 2018.

[6] S. Popli, R. K. Jha, and S. Jain, "A survey on energy efficient narrowband Internet of things (NBIoT): architecture, application and challenges," IEEE Access, vol. 7, pp. 16739-16776, 2019.

[7] G. Han, L. Wan, J. J. P. C. Rodrigues, H. Wu, and D. Zhang, "IEEE access special section editorial: green communications and networking for 5G," IEEE Access, vol. 6, pp. 7926379271, 2018. 
[8] N. Panwar, S. Sharma, and A. K. Singh, "A survey on 5G: the next generation of mobile communication," Physical Communication, vol. 18, pp. 64-84, 2016.

[9] H. Ullah, N. Gopalakrishnan Nair, A. Moore, C. Nugent, P. Muschamp, and M. Cuevas, " $5 \mathrm{G}$ communication: an overview of vehicle-to-everything, drones, and healthcare usecases," IEEE Access, vol. 7, pp. 37251-37268, 2019.

[10] G. J. Foschini and Z. Miljanic, "A simple distributed autonomous power control algorithm and its convergence," IEEE Transactions on Vehicular Technology, vol. 42, no. 4, pp. 641-646, 1993.

[11] S. Gupta, R. D. Yates, and C. Rose, "Soft dropping power control - a power control backoff strategy," in IEEE International Conference on Personal Wireless Communications, pp. 210214, Mumbai, India, India, Dec. 1997.

[12] M. G. d. S. Rêgo, T. F. Maciel, H. d. H. M. Barros, F. R. P. Cavalcanti, and G. Fodor, "Performance analysis of power control for device-to-device communication in cellular MIMO systems," in International Symposium on Wireless Communication Systems, pp. 336-340, Paris, France, Aug. 2012.

[13] S. Buzzi and D. Saturnino, "A game-theoretic approach to energy efficient power control and receiver design in cognitive CDMA wireless networks," IEEE Journal of Selected Topics in Signal Processing, vol. 5, no. 1, pp. 137-150, 2011.

[14] C. Shi, F. Wang, M. Sellathurai, and J. Zhou, "Non-cooperative game theoretic power allocation strategy for distributed multiple-radar architecture in a spectrum sharing environment," IEEE Access, vol. 6, pp. 17787-17800, 2018.

[15] X. Tang, P. Ren, Y. Wang, Q. Du, and L. Sun, "Efficient power control via non-cooperative target SINR competition in distributed wireless networks," in IEEE Vehicular Technology Conference, pp. 1-5, Vancouver, BC, Canada, Sep. 2014.

[16] P. Zhou, W. Liu, W. Yuan, and W. Cheng, "Energy-efficient joint power and rate control via pricing in wireless data networks," in IEEE Wireless Communications and Networking Conference, pp. 1091-1096, Las Vegas, NV, USA, Apr. 2008.

[17] D. H. Ho and T. A. Gulliver, "Prioritised and selective power control in cellular wireless networks," IET Communications, vol. 13, no. 4, pp. 433-441, 2019.

[18] S. Kandukuri and S. Boyd, "Optimal power control in interference-limited fading wireless channels with outageprobability specifications," IEEE Transactions on Wireless Communications, vol. 1, no. 1, pp. 46-55, 2002.

[19] S. F. Chien, T. O. Ting, X.-S. Yang, and K. Takahashi, "Mathematical analysis of energy efficiency optimality in multi-user OFDM systems," Wireless Communications and Mobile Computing, vol. 16, no. 3, p. 263, 2016.

[20] M. C. Gursoy, H. V. Poor, and S. Verdu, "The noncoherent Rician fading channel-part I: structure of the capacityachieving input," IEEE Transactions on Wireless Communications, vol. 4, no. 5, pp. 2193-2206, 2005.

[21] X. Leturc, P. Ciblat, and C. J. Le Martret, "Energy efficient resource allocation for type-I HARQ under the Rician channel," IEEE Transactions on Wireless Communications, vol. 18, no. 7, pp. 3739-3751, 2019.

[22] S. K. Yoo, S. L. Cotton, P. C. Sofotasios, and S. Freear, "Shadowed fading in indoor off-body communication channels: a statistical characterization using the $\kappa-\mu$ /gamma composite fading model," IEEE Transactions on Wireless Communications, vol. 15, no. 8, pp. 5231-5244, 2016.
[23] S. Büyükçorak, M. Vural, and G. K. Kurt, "Lognormal mixture shadowing," IEEE Transactions on Vehicular Technology, vol. 64, no. 10, pp. 4386-4398, 2015.

[24] T. Rappaport, Wireless Communications: Principles and Practice, Prentice Hall, Upper Saddle River, NJ, USA, 2001.

[25] J. D. Parsons, The Mobile Radio Propagation Channel, Wiley, New York, NY, USA, 2000.

[26] A. Goldsmith, Wireless Communications, Cambridge University Press, New York, NY, USA, 2005.

[27] Y. H. Lee and Y. Bar-Ness, "Power adaptation for BPSK signaling with average and peak power constraints in Rayleigh fading channels," IEEE Transactions on Communications, vol. 51, no. 11, pp. 1871-1876, 2003.

[28] P. A. Dighe, R. K. Mallik, and S. S. Jamuar, "Analysis of transmit-receive diversity in Rayleigh fading," IEEE Transactions on Communications, vol. 51, no. 4, pp. 694-703, 2003.

[29] N. Simmons, C. R. N. da Silva, S. L. Cotton, P. C. Sofotasios, and M. D. Yacoub, "Double shadowing the Rician fading model," IEEE Wireless Communications Letters, vol. 8, no. 2, pp. 344-347, 2019.

[30] Z. Sheng, H. D. Tuan, A. A. Nasir, T. Q. Duong, and H. V. Poor, "Power allocation for energy efficiency and secrecy of wireless interference networks," IEEE Transactions on Wireless Communications, vol. 17, no. 6, pp. 3737-3751, 2018.

[31] Y. Li, W. Jia, B. Cao, C. Wang, and M. Daneshmand, "Energyefficient cluster division for multi-cell joint transmission technology," Wireless Communications and Mobile Computing, vol. 16, no. 17, pp. 3045-3055, 2016.

[32] T. T. Tjhung, C. C. Chai, and X. Dong, "Outage probability for lognormal-shadowed Rician channels," IEEE Transactions on Vehicular Technology, vol. 46, no. 2, pp. 400-407, 1997.

[33] J. R. Haug and D. R. Ucci, "Outage probability of microcellular radio systems in a Rayleigh/Rician fading environment," in IEEE International Conference on Communicationspp. 306310, Chicago, IL USA, Jun. 1992.

[34] M. Rao, F. J. Lopez-Martinez, M. Alouini, and A. Goldsmith, "MGF approach to the analysis of generalized two ray fading models," IEEE Transactions on Wireless Communications, vol. 14, no. 5, pp. 2548-2561, 2015.

[35] N. Bhargav, C. R. N. da Silva, Y. J. Chun, E. J. Leonardo, S. L. Cotton, and M. D. Yacoub, "On the product of two $\kappa-\mu$ random variables and its application to double and composite fading channels," IEEE Transactions on Wireless Communications, vol. 17, no. 4, pp. 2457-2470, 2018.

[36] O. S. Badarneh, T. Aldalgamouni, and M. Aloqlah, "Outage probability analysis of multi-hop relayed wireless networks over $\eta-\mu$ fading channels," AEU - International Journal of Electronics and Communications, vol. 67, no. 10, pp. 833838, 2013.

[37] G. G. Roussas, "Exponential probability inequalities with some applications," in IMS Lecture Notes, Statistics, Probability and Game Theory, vol. 30, pp. 303-319, 1996.

[38] J. Steele, The Cauchy-Schwarz Master Class: An Introduction to the Art of Mathematical Inequalities, Cambridge University Press, Cambridge, UK, 2013.

[39] D. S. Mitrinović, J. E. Pecărić, and A. M. Fink, Classical and New Inequalities in Analysis, Springer, Berlin, 1993.

[40] T. J. L'. A. Bromwich, An Introduction to the Theory of Infinite Series, Macmillan, London, OK, 1959. 
[41] N. Lord, "92.73 On inequalities equivalent to the inequality of the means," The Mathematical Gazette, vol. 92, no. 525, pp. 529-533, 2008.

[42] M. Chiang, C. W. Tan, D. P. Palomar, D. O'Neill, and D. Julian, "Power control by geometric programming," IEEE Transactions on Wireless Communications, vol. 6, no. 7, pp. 2640-2651, 2007.

[43] S. Fu, H. Wen, and B. Wu, "Power-fractionizing mechanism: achieving joint user scheduling and power allocation via geometric programming," IEEE Transactions on Vehicular Technology, vol. 67, no. 3, pp. 2025-2034, 2018.

[44] N. C. Beaulieu and Q. Xie, "An optimal lognormal approximation to lognormal sum distributions," IEEE Transactions on Vehicular Technology, vol. 53, no. 2, pp. 479-489, 2004.

[45] J. Chen, L.-C. Wang, and C.-H. Liu, "Coverage probability of small cell networks with composite fading and shadowing," in IEEE International Symposium on Personal, Indoor, and Mobile Radio Communication, pp. 1965-1969, Washington, DC, Sep. 2014.

[46] B. Dulek, N. D. Vanli, S. Gezici, and P. K. Varshney, "Optimum power randomization for the minimization of outage probability," IEEE Transactions on Wireless Communications, vol. 12, no. 9, pp. 4627-4637, 2013.

[47] K. Yang, S. Martin, D. Quadri, J. Wu, and G. Feng, "Energyefficient downlink resource allocation in heterogeneous OFDMA networks," IEEE Transactions on Vehicular Technology, vol. 66, no. 6, pp. 5086-5098, 2017.

[48] A. Zappone, L. Sanguinetti, and M. Debbah, "Energy-delay efficient power control in wireless networks," IEEE Transactions on Communications, vol. 66, no. 1, pp. 418-431, 2018. 